\title{
Es besteht Bedarf für neue Substanzen
}

„Jeder vierte Patient mit einer Colitis ulcerosa (CU) zeigt einen chronisch anhaltenden Verlauf“, erläuterte Prof. Peter Irving, Gastroenterologe, London Clinic, Großbritannien. Auch erforderten 20 - 30 \% innerhalb der ersten zehn Jahre nach der Diagnosestellung eine Kolektomie, wobei die Notwendigkeit für einen solchen Eingriff in den letzen Jahrzehnten deutlich abgenommen habe. Doch trotz gewisser Fortschritte seien auch heute noch viele Patienten unzureichend therapiert. Folgen sind strukturelle Veränderungen am Darm, die zu Tenesmen und Inkontinenz führen können, und die Entartung. „Das Risiko für ein Karzinom steigt mit der Krankheitsdauer und der histologischen respektive endoskopischen Schwere der Entzündung“, betonte Irving.

Im Allgemeinen erfolgt eine Step-upTherapie. Bei leichten bis moderaten Verläufen wird mit Aminosalizylaten und
Steroiden begonnen, bei Steroidrefraktärität oder -abhängigkeit kommen Immunsuppressiva zum Einsatz, um die Remission zu erhalten, und bei schweren Verläufen sind Biologika indiziert. „Doch die TNF-alpha-Inhibitoren sind mit einer Reihe von Problemen behaftet", erklärte Irving. Dazu gehört das primäre Therapieversagen bei $>20 \%$. Bei weiteren $20 \%$ nimmt die Wirksamkeit unter der Therapie ab, wofür die Bildung von Antikörpern gegen das Medikament verantwortlich ist. Die Umstellung auf eine andere Substanz löst das Problem meist nicht. Nicht selten müsse der TNF-alpha-Inhibitor auch wegen Nebenwirkungen abgesetzt werden.

„Dies alles führt dazu, dass bei vielen Patienten die Therapieziele nicht erreicht werden“, so Irving. Gefordert werde heute nicht nur eine klinische und endoskopische Remission, sondern auch eine his- tologische, also eine mukosale Heilung. Eine solche beeinflusse den Krankheitsverlauf günstig, das heißt es treten weniger Rezidive auf und die Notwendigkeit für eine stationäre Behandlung oder Operation wird gesenkt. Es bestehe Bedarf für neue Therapiestrategien, um diese Ziele zu erreichen. Zu den in Entwicklung befindlichen Substanzen gehört der Janus-Kinase-Inhibitor Tofacitinib. Die Hemmung der Januskinasen (JAK) ist ein neues vielversprechendes Therapiekonzept. Die Substanz kann oral gegeben werden und verspricht eine dauerhaft anhaltende Wirkung, da die Substanz nicht immunogen wirkt. Im Unterschied $\mathrm{zu}$ den Biologika werden verschiedene pathogenetische Mechanismen, die bei der Pathogenese der Erkrankung beteiligt sind, gemeinsam gehemmt.

Dr. med. Peter Stiefelhagen

Satellitensymposium „Pharmacological and clinical perspectives: The evolution of the treatment landscape and personalised medicine approaches for management of UC" UEG Week, 30.11.2017, Barcelona; Veranstalter: Pfizer

\section{Gastrointestinale Tumoren}

\section{Therapiesequenz immer weiter präzisieren}

Bei Patienten mit metastasiertem Kolorektalkarzinom (mCRC) könnte sich die Therapie an verschiedenen etablierten oder zumindest identifizierten Parametern, wie bestimmten Biomarkern, der Tumorlokalisation und der Pharmakokinetik, orientieren. Auch die Behandlung von Patienten mit metastasiertem $\mathrm{Ma}$ genkarzinom wird in diesem Sinn zusehends zielgerichteter. Unabhängig davon gilt es, eine Mangelernährung der Patienten als klinisch hoch relevanten, unabhängigen Risikofaktor zu vermeiden.

Einen möglichen Therapiealgorithmus für mCRC-Patienten stellte Prof. Stefan Kasper, Onkologe mit dem Schwerpunkt gastrointestinale Onkologie, Universitätsklinikum Essen, vor: Ausgehend vom molekularen Profil ergibt sich jeweils eine Therapiesequenz für das RAS-Wildtyp-, das RAS-mutierte oder das BRAFmutierte mCRC, wobei beim RAS-Wildtyp-mCRC nochmals nach der Tumorlokalisation unterschieden wird. Bei linksseitig gelegenem Primarius wird in der
Erstlinientherapie mit einer Anti-EGFR (Epidermal Growth Factor Receptor)basierten Kombination begonnen, bei rechtsseitig gelegenem Primarius ebenso wie generell bei RAS-mutiertem mCRC mit einer Bevacizumab-basierten Kombination. In der Zweitlinientherapie folgt darauf jeweils eine Anti-VEGF (Vascular Endothelial Growth Factor)-basierte Kombination. Welches der verfügbaren antiangiogenen Wirkungsprinzipien wird aber eingesetzt? Ist die von Kasper erläuterte Beobachtung, dass sich die pro-angiogenen Faktoren unter einer Angiogeneseinhibition in der Erstlinie etwas verändern können, relevant? Wäre also ein etwas breiterer Ansatz günstig?

\section{mCRC: Angiogeneseinhibition mit} Ramucirumab

Der Antikörper Ramucirumab (Cyram$\mathrm{za}^{\varpi}$ ) ist gegen den VEGF (Vascular Endothelial Growth Factor)-Rezeptor 2, einen für die Tumorangiogenese zentralen Mediator, gerichtet. Ramuciruamb kann entsprechend der Zulassung in Kombination mit FOLFIRI (Folinsäure/5-Fluorouracil/Irinotecan) bei mit Bevacizumab, Oxaliplatin und einem Fluoropyrimidin vorbehandelten Patienten eingesetzt werden. In dieser Patientengruppe wurde in der Phase-III-Studie RAISE im Vergleich zu Placebo plus FOLFIRI ein statistisch signifikanter Zugewinn beim Gesamtüberleben (OS) gezeigt (primärer Endpunkt: Hazard Ratio [HR] 0,844; $95 \%$-Konfidenzintervall [95\%-KI] 0,730 0,$976 ; p=0,0219$ ) [Tabernero J et al. Lancet Oncol 2015; 16: 499 - 508]. In ergänzenden Analysen konnten zwei Parameter identifiziert werden, die als On-treatment- oder prädiktiver Marker dienen könnten: eine hohe Ramucirumab-Plasmakonzentration unter der Therapie und eine hohe VEGF-D-Plasmakonzentration in der Ausgangssituation [Cohn AL et al., Cancer Chemother Pharmacol 2017 ,Epub ahead of print, Tabernero J et al., Ann Oncol 2017; 28 (suppl_5): 555P].

Dr. Annemarie Burgemeister

Symposium „Therapie von Gastrointestinalen Tumoren: Der Patient im Mittelpunkt", DGHO-Jahrestagung 2017, 29.9.2017, Stuttgart; Veranstalter: Lilly Deutschland 